\title{
An Approach to Medical Image Compression Using Filters Based On Lifting Scheme
}

\author{
Anju B ${ }^{1}$,Manimurugan $\mathrm{S}^{2}$
}

\begin{abstract}
Image compression plays an important role in the compression of medical images. Medical imagings are mainly used for diagnosis of diseases and surgical planning. Medical images are usually stored digitally. Medical Image compression plays an important role in telematics especially in telemedicine. It is necessary that medical images need to be compressed for reliability to be transmitted. In the medical image compression diagnosis is effective only when the compressed image preserves all the information of the original image. This results in a lossless compression technique. While lossy compression techniques, are more efficient in terms of storage and transmission needs but there is no warranty that they can preserve the characteristics needed in medical image processing and diagnosis. Compression plays an important role as medical imaging moves to film less imaging. CTI or MRI medical imaging are used nowadays which can produce pictures of the human body in digital form. The lifting scheme is used for the design of both orthogonal and bi-orthogonal filters .It is implemented for the orthogonal filters using two lifting steps. The performance of the proposed filters are then compared with the conventional filters in terms of compression ratio, PSNR etc. The bi-orthogonal filters are implemented using the SPIHT algorithm replacing the EZW coding for image compression. SPIHT algorithm requires fewer bits to capture the same amount of information when compared with EZW proposed.
\end{abstract}

Keywords: Bi-orthogonal wavelet transforms, Lifting scheme, SPIHT algorithm, EZW coding, orthogonal filters.

\section{Introduction}

Medical image compression plays a key role in hospitals which moves towards filmless imaging and results in digital imaging. This is true in the case of grayscale images that are used in radiology applications. The images may be very large in size and number and compression is done to reduce the cost of storage and ease of transmission. It is a fact that medical images are increasingly acquired in digital format. The major imaging modalities include Computed Tomography (CT), Magnetic Resonance Imaging (MRI), Ultra Sonography (US), Positron Emission Tomography (PET), Single Photon Emission Computerized Tomography (SPECT), Nuclear Medicine (NM), Digital Subtraction Angiography (DSA) and Digital Flurography (DF) [18]. All these techniques have made available the view of cross-sections of the human body, permitted to navigate inside it and to design novel minimally invasive techniques to investigate many pathologies. Radiology is undergoing a profound transition from interpretation of images displayed on film to reading images on highresolution computer monitor screens. As this new era emerges, radiologists are also starting to see traditional film delivery replaced by electronic transfer of digital files, and film storage rooms replaced by archives of computer files. The medical enterprise depends on a system that makes diagnostic images available for radiologic interpretation, that transmits images to physicians throughout the system, and that efficiently stores images pending retrieval for future medical or legal purposes. Computerized medical imaging generates large, data-rich electronic files. To speed electronic transfer and minimize computer storage space, medical images often undergo compression into smaller digital files. The level of diagnostic detail needed for clinical interpretation of medical images varies according to modality. In general, nuclear medicine scans require less detail than computed tomography (CT) or magnetic resonance (MR)[18-20]. Because interpretation of mammography and radiography depends on high spatial resolution, these images demand more detail than CT or MR, which need high contrast resolution for diagnostic interpretation. The need for compressing medical images:

- Growing need for storage

- Efficient data transmission

- Telemedicine

- Tele-radiology applications

- Real time Tele-consultation

- PACS (Picture archiving and communication systems)

There are several forms of filters for image compression. The choice of the filters in the compression is an issue that affects the quality of the system. Hybrid filters also exist to maximize compression performance. Here we are trying to use a new type of bi-orthogonal filters by taking into advantage the cosine modulation of both analysis synthesis filters by considering FIR-IIR filters [1].Results indicate that linear phase Bi-orthogonal 
filters have better compression performance than the orthogonal filters which don't have a linear phase. When the wavelet transforms uses linear phase filters it provides symmetric outputs when symmetric inputs are given. Evaluation can be done reliably by considering m-level analysis synthesis system with a single input and single output. Cosine modulation is done to simplify the design and to increase the efficiency. Here we are taking into consideration the analysis prototype as an IIR filter with FIR filter as a particular case. In the case of orthogonal filters with m-channel, one analysis filter is arbitrary and rest of the filters including the analysis and the synthesis filters are parameterized.

The lifting scheme for bi-orthogonal wavelet transform consists of split, predict, update [2].This is an efficient scheme when considering the second generation wavelets. The lifting scheme has the advantages such as faster implementation, reversible integer-integer transforms etc. The lifting has been adopted in several standards including JPEG2000 standard [3]. JPEG 2000 compress images based on the wavelet transform. Linear phase FIR bi-orthogonal filters have been popularly in use for the JPEG standard. The EZW coding is adopted as the standard in JPEG. In orthogonal filters the lifting scheme is implemented using two lifting steps. This states that there is more rounding errors. IIR orthogonal filters use allpass filters in the lifting scheme. The filter with fewer lifting tends to perform better than others. Every wavelet transform with the FIR filters can be decomposed into finite number of lifting steps. But it is not possible in the case of IIR filters to be decomposed into a finite number of lifting steps.

The discrete wavelet transform has been extensively used in digital and signal image processing. Both the orthogonality and symmetry of the wavelets are important for many applications. Symmetry requires all the filters to be linear phase. Various classes of orthogonal with approximate linear phases and bi-orthogonal filters with exactly linear phases are proposed using FIR and IIR filters. In the sub band/wavelet image coding [9] used for decomposition was size limited to avoid increasing of the number of samples to be coded. Such coding is used in the JPEG standard. This compression method included three sections in which the first included a subbands section where image is decomposed into subbands using analysis filters and the second section included called quantization/coding where subbands are first quantized and later coded for transmission and the third section called synthesis where quantized subbands are reconstructed into a full image using synthesis filter. The analysis and synthesis filters are essential for the subband/wavelet [7] compression algorithm. The image decomposition can be done to obtain the horizontal orientation sub image, vertical orientation sub image, diagonal orientation sub image and the approximation sub image where the image is subdivided again into four subsections. The FIR (Finite Impulse Response) and IIR (Infinite Impulse Response) are filters where the output is finite a certain number of times in the FIR and infinite in the case of IIR. The output is finite for the FIR due to the feedback present in it.

In the subsequent sections it includes the description of a bi-orthogonal filter by taking into advantage of the FIR and IIR filters. The lifting scheme has been adopted for the orthogonal filters where the number lifting steps is more when compared with the bi-orthogonal filters. The performance of the filter is determined using the compression ratio, PSNR value etc. Higher compression ratio provides better quality images.

\section{Proposed System}

In this paper image compression is performed using bi-orthogonal filters where the compression is done using wavelet decomposition .The decomposed images are then passed through an $\mathrm{m}$ channel analysis synthesis filter and the compression is done using FIR-IIR filters[8-11].This is analyzed to get the horizontal ,vertical, approximation and diagonal details. The result is a lossless compression image. The analysis-synthesis filters are based on the Quadrature Mirror Filters (QMF) [9].

Decompression can be done for the same image by transmitting the same to an inverse wavelet decomposition to determine how the image is similar when compared with the original image. This results in a lossless compression where the reconstruction condition is applied to perform the reconstruction.

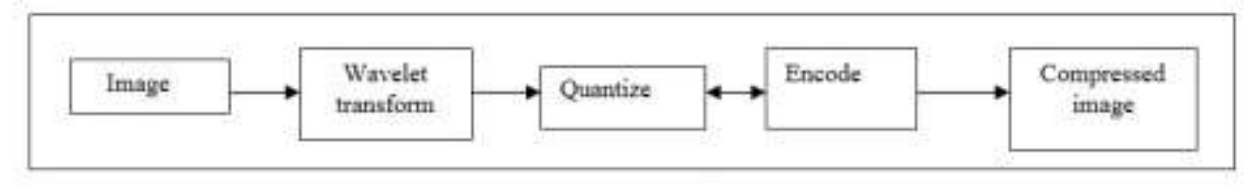

Fig 2.1. Compression of image

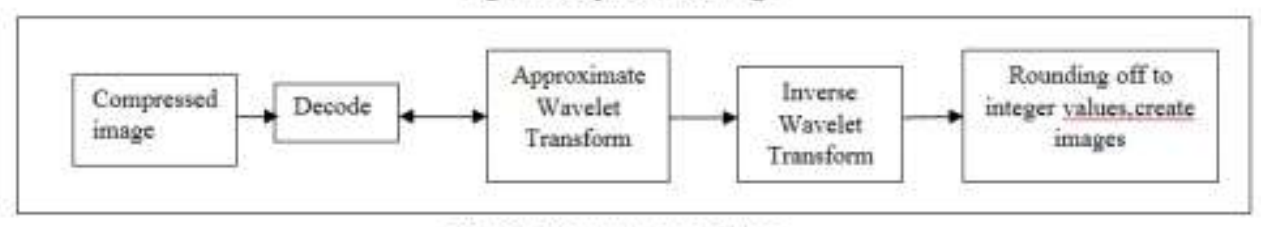

Fig 2.2. Decompression of image 
The five stages of compression and decompression are shown in the following figures. All the steps described are invertible except for one step quantize and hence it is a lossless compression. Quantizing refers to a reduction of the precision of the floating point values of the wavelet transform, which are typically either 32-bit or 64-bit floating point numbers. To use lower number of bits in the compressed transform which is necessary if compression of $8 \mathrm{bpp}$ or $12 \mathrm{bpp}$ images is to be achieved these transform values must be expressed with less number of bits for each value. This may lead to errors. When an inverse transform is performed these approximate, quantized, wavelet transforms will produce approximations to the images. This create an error inherent in lossy compression.

The lifting scheme used in the proposed method is an efficient tool for constructing second generation wavelets.It haas been adopted in the international standard JPEG 2000.It has the advantages such as faster implementation,fully inplace calculation,reversible integer-to-integer transforms etc.IIR filters cannot be decomposed into a finite number of lifting steps.

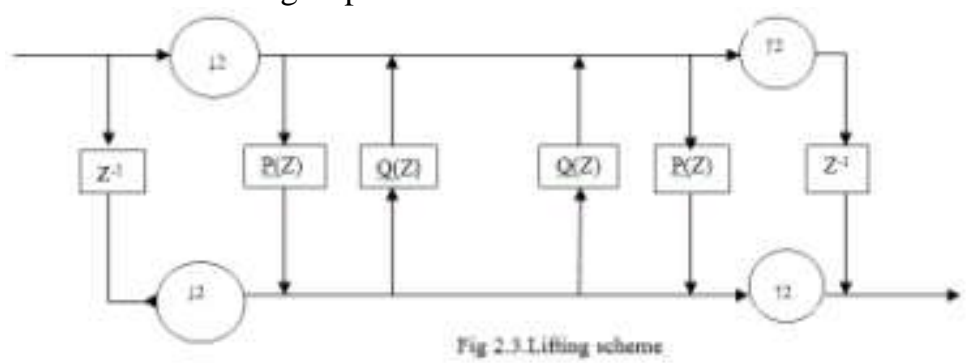

In the figure 3 sub filter $\mathrm{p}(\mathrm{z})$ is a prediction operator and $\mathrm{q}(\mathrm{z})$ is an update operator. This is attractive in lossless coding applications where the original image can be reconstructed from the compressed image without losing the quality. Let $\mathrm{M}_{0}(\mathrm{z})$ and $\mathrm{M}_{1}(\mathrm{z})$ be a pair of low pass and high pass filters in the analysis bank. Their transfer functions denoted by $\mathrm{M}_{0}$ and $\mathrm{M}_{1}(\mathrm{z})$ are given by

$$
\begin{aligned}
M_{0}(z) & =1+Q\left(z^{2}\right) M_{1}(z) \\
M_{1}(z) & =z^{-1}-P\left(z^{2}\right)
\end{aligned}
$$

Therefore, the design problem of the filter bank $\mathrm{H}_{0}(\mathrm{z})$ and $\mathrm{H}_{1}(\mathrm{z})$ becomes how to determine two transfer functions $\mathrm{P}(\mathrm{z})$ and $\mathrm{Q}(\mathrm{z})$ to meet some of the design specification. In the design of $\mathrm{P}(\mathrm{z})$ and $\mathrm{Q}(\mathrm{z})$ FIR filters are often used so far to get a class of bi-orthogonal wavelet filter banks with exact linear phase while IIR filters have been also used to design a class of bi-orthogonal wavelet filter banks with the causal stability or with exact linear phase.

Algorithm

In this paper we use the SPIHT algorithm .In SPIHT it requires few bits to capture the same amount of information. By using SPIHT [6] algorithm where the original image can be defined by a set of pixels $\mathrm{P}_{\mathrm{j}, \mathrm{k}}$ where $(\mathrm{j}, \mathrm{k})$ is the pixel coordinate. Each element $\mathrm{b}_{\mathrm{j}, \mathrm{k}}$ is called transform coefficient at each coordinate $(\mathrm{i}, \mathrm{j})[6]$ The algorithm is described as follows [12].SPIHT algorithm provides description of 3 sets of indices.

$\mathrm{B}(\mathrm{n})=\{$ Descendent indices of the index $\mathrm{m}\}$

$\mathrm{E}(\mathrm{n})=\{$ Child indices of the index $\mathrm{m}\}$

$\mathrm{H}(\mathrm{n})=\mathrm{B}(\mathrm{n})-\mathrm{E}(\mathrm{n})=\{$ Grandchildren of $n$, i.e., descendants which are not children of $\mathrm{n}\}$

SPIHT provides three lists for the set of indices such as list of insignificant sets (LIS), the list of insignificant pixels (LIP), and the list of significant pixels (LSP). An index $\mathrm{n}$ is called either significant or insignificant, depending on whether the transform value $\mathrm{w}(\mathrm{n})$ is significant or insignificant with respect to a given threshold. For the LIS, the index $\mathrm{m}$ denotes either $\mathrm{B}(\mathrm{n})$ or $\mathrm{H}(\mathrm{m})$. In the former case, the index $\mathrm{m}$ is said to be of type $\mathrm{B}$ and, in the latter case, of type $\mathrm{H}$.

Step 1 (Initialize).

First select an initial threshold V0.

All transform values satisfy $\mathrm{w}(\mathrm{n})<\mathrm{V} 0$.

At least one value should satisfy $\mathrm{w}(\mathrm{n}) \geq \mathrm{V} 0 / 2$.

Let $\mathrm{LIP}=\mathrm{M}$,

$\mathrm{LSP}=\dot{\varnothing}$, and

LIS $=$ all the indices in $\mathrm{M}$ that have descendants (assigning them all type B).

Step 2 (Update threshold):Let $\mathrm{Vs}_{\mathrm{s}}=\mathrm{V}_{\mathrm{s}-1} / 2$.

Step 3 (Sorting pass) $\mathrm{m}=0$;

$$
\mathrm{m} \leq \max [\mathrm{LSP}] ; \mathrm{m}++
$$

Print" $S_{k}(m) ;$ 


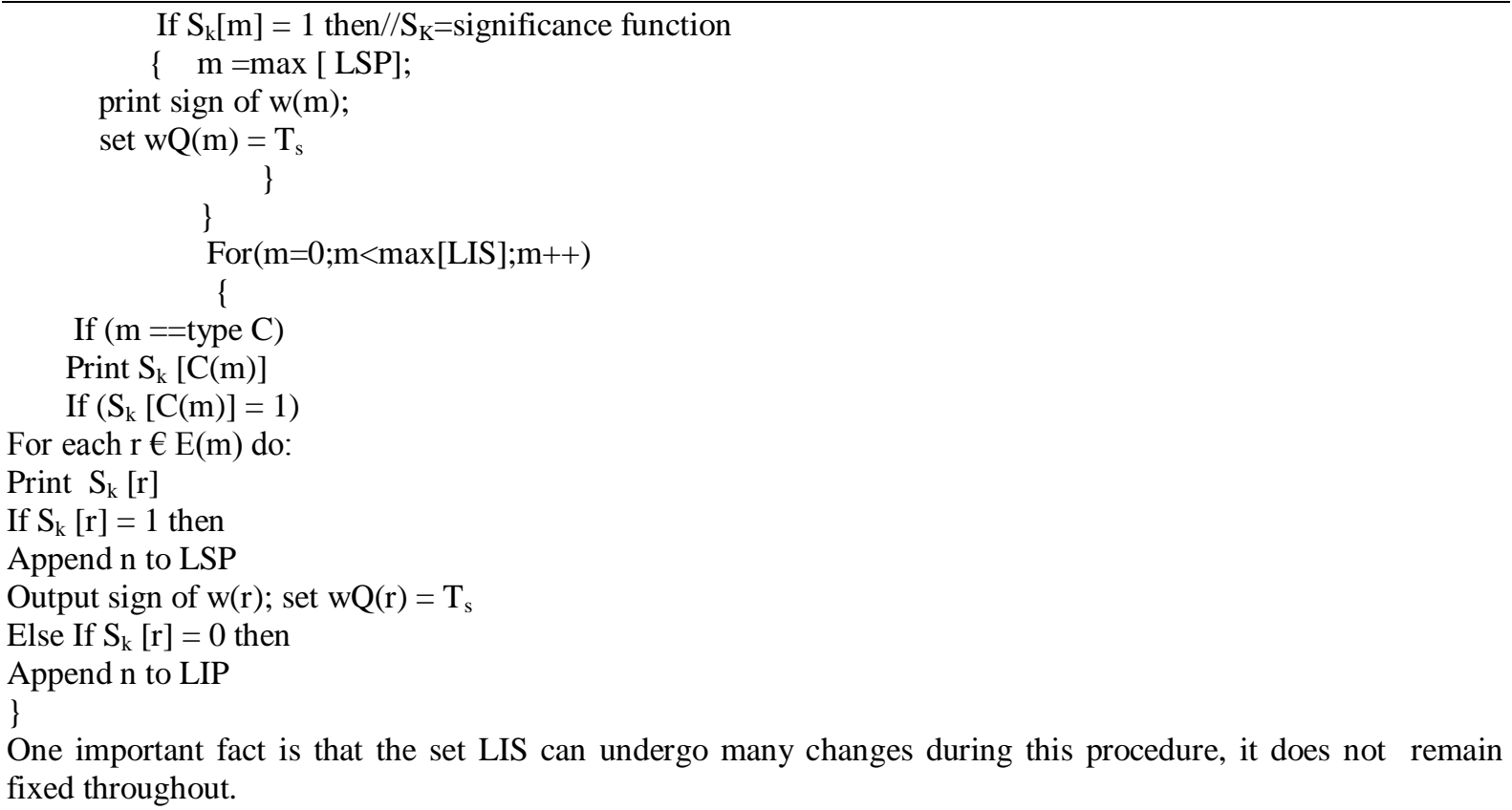

Step 4 (Refinement pass):Scan through indices $m$ in LSP found with higher 20 threshold values $\mathrm{Tj}$, for $\mathrm{j}<\mathrm{k}$ (if $\mathrm{k}=1$ skip this step).

Step 5 (Loop): Repeat steps from 2 to 4.

\section{Results And Discussion}

In this paper three test images are taken which are gray scale images. We have taken into consideration the original size of the image, compressed and decompressed size. The decompressed size is approximately equal to the size of original image. Time is considered a factor during the compression. The size is taken in Kilo Bytes(KB) and the time in seconds(s).The time for compression as well as decompression are compared. Using these data the compression ratio(CR),Mean square error(MSE) and the Peak Signal to Noise Ratio(PSNR) are calculated. The $\mathrm{CR}$ is calculated using the formula,

$\mathrm{CR}=1\left(-\frac{\text { size of compressed image }}{\text { Size of original image }}\right) * 100$

The Mean Squared Error takes the values ranging from 1-4.

The PSNR values can be calculated using the MSE obtained

$\mathrm{PSNR}=20 \log _{10}\left(\frac{255}{\sqrt{\mathrm{MSE}}}\right)$

The larger the PSNR value and the SNR value indicate a smaller difference between the original and the reconstructed image. The main advantage of this is the ease of computation but it does not reflect perceptual quality. An important property of PSNR is that a large numerical distortion can cause a slight spatial shift of an image. Conversely a small average distortion can result in a damaging visual artifact, if all the error is concentrated in a small important region. The grayscale images are taken for the calculation. Of all the quality measures PSNR is the most important measure which maintains the quality of the images approximately. The value ranges from 0-255.To calculate the PSNR value the maximum value of the grayscale images are taken which is 255 .

Many irreversible compression technique involves three steps; transformation, quantization and encoding. Transformation is a step in which image is transformed from grayscale values in spatial domain to some other values. Quantization is a step in data integrity is lost. It preserves the most important coefficients by converting the floating point values to integer values. Finally quantized coefficients are encoded during the encoding step. JPEG is a widely used compression technique that includes both reversible and irreversible techniques. Although it was not designed for medical images it is used for radiologic images. The following 
figure shows the compression of images based on JPEG. It divides the images into 8pixel X 8pixel blocks. The DCT of each image block is computed[18]. Next quantization is performed. Then the coefficients are approximated to values that are easy to represent in a small amount of space. There is an $8 \times 8$ table which contains the values by which the coefficients are divided. The resultant values are rounded to the nearest integers. The JPEG2000 effort was to be developed. This was introduced to overcome the shortcomings of the JPEG standard. Some of these are:

- Better performance at higher compression ratios

- A single code stream that would support irreversible and lossless compression.

- Support for many types of images

- Support for many different environments (high performance local area network or low-speed wide area network.

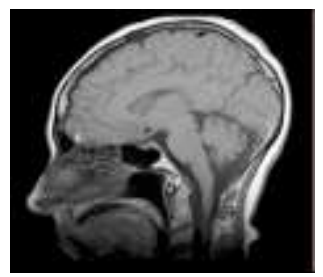

MRI / FMRI (Function Magnetic Resonance)

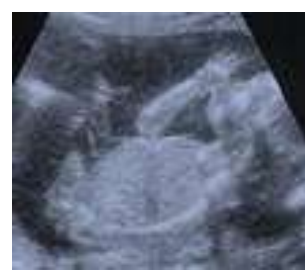

Dynamic 3D Ultrasound

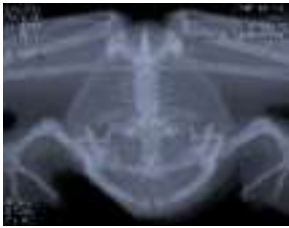

CT (computerized Tomograhpy)

Fig 3.1. Some examples of medical images

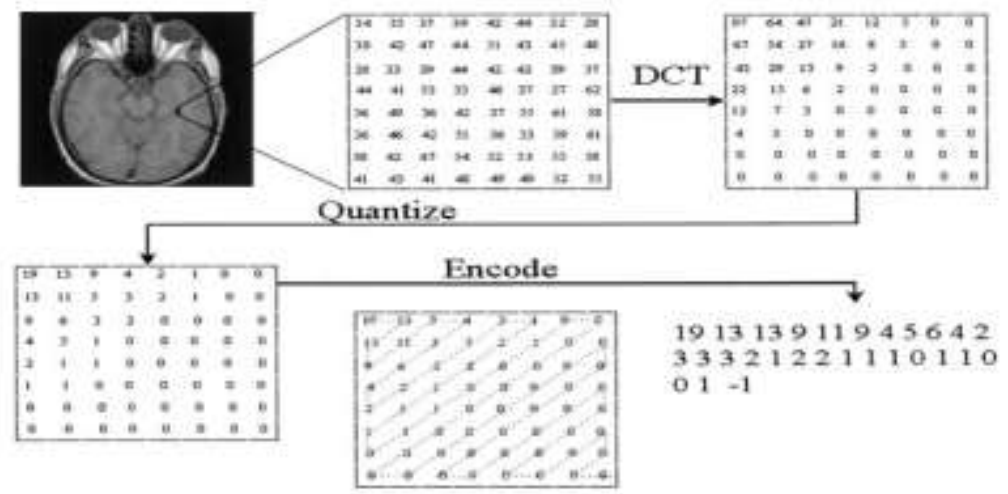

Fig 3.2.JPEG algorithm. The image is first separated into $8 \times 8$ pixel subimages. The DCT of each subimage then is computed. These coefficients are then quantized using the quantization table. Finally the quantized values are encoded from the upper left corner with a marker value sent when here are no more non zero values.

Fig 3.1 shows the different test images taken and determining their compression performances using various measures.

Table 3.1 Comparison of size of test images

\begin{tabular}{|l|c|c|c|c|c|}
\hline \multirow{2}{*}{ Name of image } & \multicolumn{3}{|c|}{ Size(KB) } & \multicolumn{2}{c|}{ Time(s) } \\
\cline { 2 - 6 } & $\begin{array}{c}\text { Original } \\
\text { size }\end{array}$ & $\begin{array}{c}\text { Compressed } \\
\text { size }\end{array}$ & $\begin{array}{c}\text { Decompressed } \\
\text { size }\end{array}$ & Compression & Decompression \\
\hline MRI/FMRI & $148 \mathrm{~KB}$ & $64.0 \mathrm{~KB}$ & $148.2 \mathrm{~KB}$ & 7.06 & 6.1 \\
\hline Ultrasound & $184 \mathrm{~KB}$ & $89.27 \mathrm{~KB}$ & $184.1 \mathrm{~KB}$ & 5.79 & 5.31 \\
\hline $\mathrm{CT}$ & $40.0 \mathrm{~KB}$ & $17.24 \mathrm{~KB}$ & $41.5 \mathrm{~KB}$ & 4.25 & 4.0 \\
\hline
\end{tabular}

From the table 3.1.it is clear that the size of the compressed image is less than the size of the original image. The decompressed size is slightly greater than or less than those of the original image. The time for the compression and the decompression are considered. The time for decompression is less than the time for compression.

Table 3.2.Comparison of performance measures of different images 


\begin{tabular}{|l|c|c|c|}
\hline Name of image & CR & MSE & PSNR \\
\hline MRI/FMRI & 56.75 & 1.6 & 46.12 \\
\hline Ultrasound & 51.48 & 2.3 & 44.55 \\
\hline CT & 56.9 & 3.8 & 42.37 \\
\hline
\end{tabular}

The table 3.2 compares the various test images with the various CR,MSE,PSNR calculated. The MSE values ranges from 1 to 4 . Generally, when the PSNR is $40 \mathrm{~dB}$ or larger, then the two images are virtually indistinguishable by human observers.

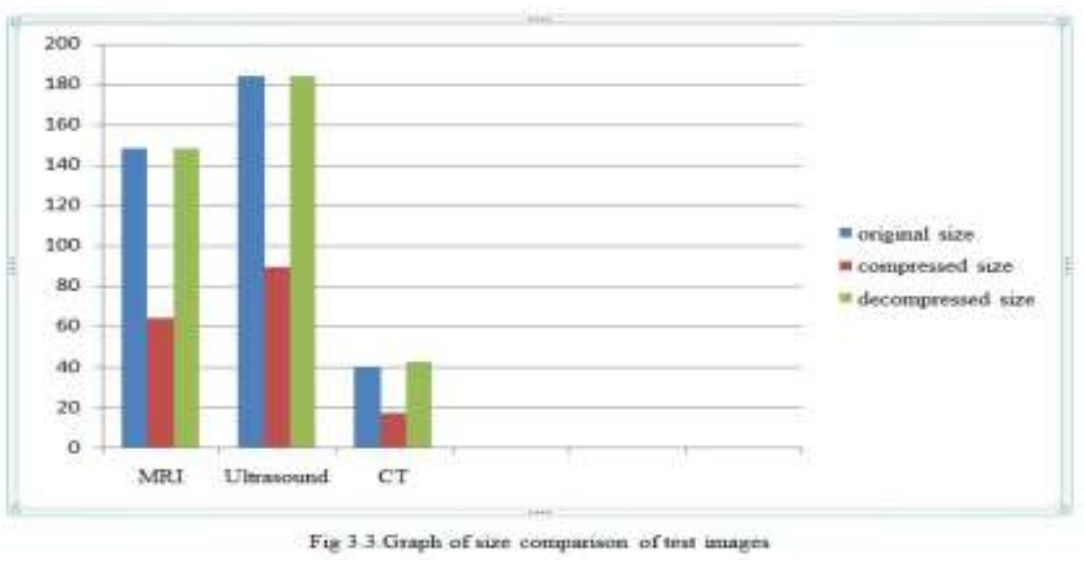

Fig.3.3 shows the different images with their sizes. The original size, compressed size and the decompressed size of the different images are compared. The name of the images are taken along the $\mathrm{X}$-axis and the size of the images in $\mathrm{KB}$ are taken along the $\mathrm{Y}$-axis.
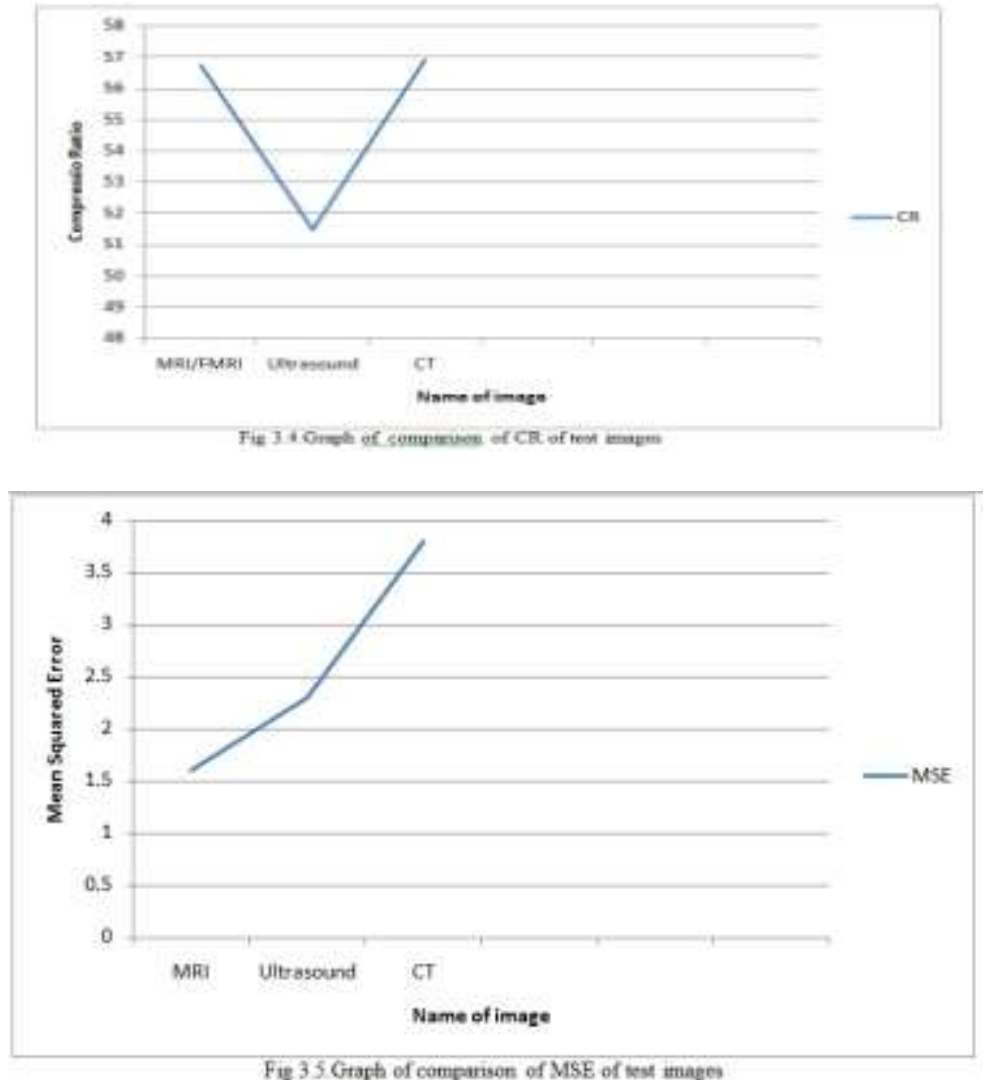

Fie 35 Graph of comparison of MSE of sest mages 


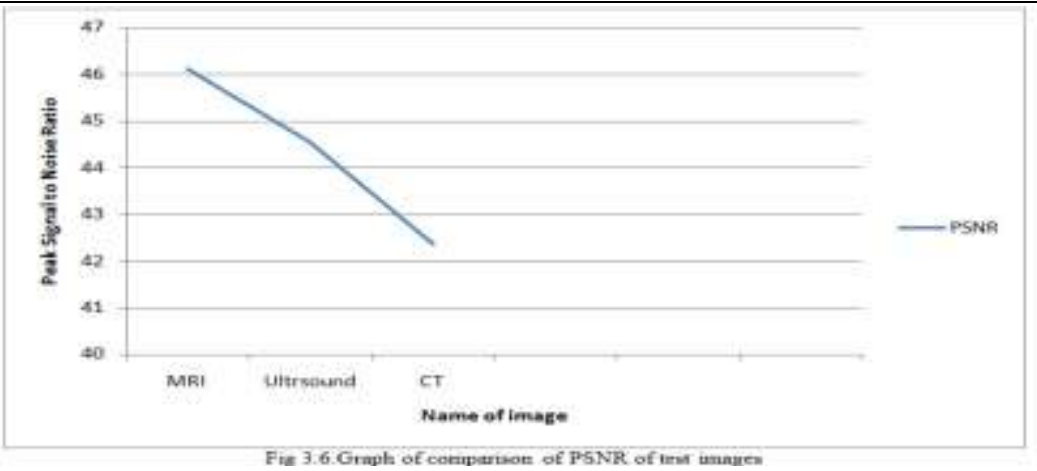

Fig 3.4,3.5,3.6 shows the different images with their CR,PSNR,MSE. The name of the images are taken along the $\mathrm{X}$ axis and the different rates of the images in $\mathrm{KB}$ are taken along the Yaxis. A small value of the MSR shows only slight variation in the original and the compressed image.

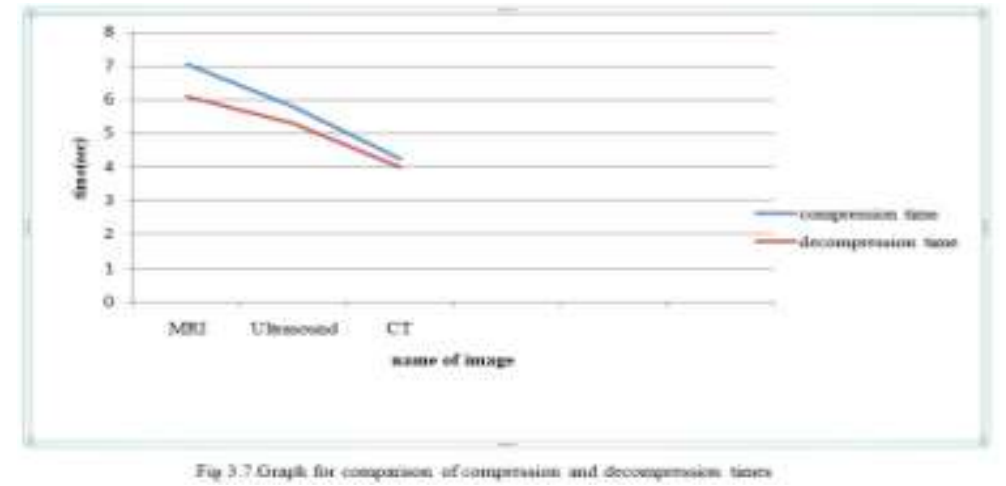

Fig.3.7 gives the comparison of the different times taken for compression as well as decompression. It is clear that the time taken for decompression is less than the time taken for compression. The different test images such as lena ,barbara etc are taken along the x-axis and the time in seconds is taken along the $y$-axis. Sometimes there may be slight variations or almost equal time between those of compression and decompression. From the system diagram described we know the time taken for compression is the time for passing through m-channel after the dyadic wavelet decomposition whereas the time for decompression is the time for inverse wavelet decomposition.

\section{Conclusion}

The main achievement of this paper is the compression of medical images using filters. It has also taken into advantage the SPIHT algorithm. One of the main features of this coding is that ordering of data is not explicitly transmitted. We need not sort all the coefficients in this algorithm. Another advantage of this is it takes into account the lifting scheme.

Here the performance of different test images are compared by finding the CR,PSNR,MSE of the images. This is done comparing with the existing bi-orthogonal filters which resulted in better compression performance with the former one .From the observations it is clear that the as the compression ratio of the images increases the quality of the image is increased as well. This differs from the earlier one where the compression ratio and quality of the image was inversely proportional. This shows a success rate of 80 percent. Also the test images maintain only slight variations between the original and the compressed image. The compression ratios are in the range of 40's and the PSNR values in the range of 50's. Thus we infer that the compression ratios and quality of the images are directly proportional when the bi-orthogonal filters are used and implemented using the lifting scheme.

\section{Acknowledgement}

The author would like to thank all the reference authors of the papers which they have taken to do their researchwork who are members of the IEEE Professional society. The author would also like to thank the anonymous reviewers, whose comments and suggestions have helped them to improve the quality of the original manuscript 


\section{References}

[1] F. Argenti and E. Del Re, "Design of biorthogonal M-channel cosine modulated FIR/IIR filter banks," IEEE Trans. Signal Process., vol. 48, no. 3, pp. 876-881, Mar. 2000.

[2] A. Z. Averbuch and V. A. Zheludev, "A new family of spline-based biorthogonal wavelet transforms and their application to image compression,” IEEE Trans. Image Process., vol. 13, no. 7, pp. 993-1007,Jul. 2004.

[3] X. Zhang, W. Wang, T. Yoshikawa, and Y. Takei, "Design of IIR orthogonal wavelet filter banks using lifting scheme," IEEE Trans. Signal Process., vol. 54, no. 7, pp. 2616-2624, Jul. 2006.

[4] J. M. Shapiro, "Embedded image coding using zerotrees of wavelets coefficients," IEEE Trans. Signal Process., vol. 41, no. 12, pp.3445-3462, Dec. 1993.

[5] J. Villasenor, B. Belzer, and J. Liao, "Wavelet filter evaluation for image compression," IEEE Trans. Image Process., vol. 4, no. 8, pp.1053-1060, Aug. 1995.

[6] A. Said and W. A. Pearlman, "A new fast and efficient image codec based on set partitioning in hierarchical trees," IEEE Trans. Circuits Syst. Video Technol., vol. 6, no. 3, pp. 243-250, May 1996.

[7] M. Antonini, M. Barlaud, P. Mathieu, and I. Daubechies, "Image coding using wavelet transform,” IEEE Trans. Image Process., vol. 1,no. 2, pp. 205-220, Apr. 1992.

[8] C. W. Kim and R. Ansari, "FIR/IIR exact reconstruction filter banks with applications to subband coding of images," in Proc. 34th Midwest Symp. Circuits Syst., May 1991, pp. 227-230.

[9] M. J. T. Smith and S. L. Eddins, “Analysis/synthesis techniques for subband image coding,” IEEE Trans. Acoust., Speech, Signal Process., vol. 38, no. 8, pp. 1446-1456, Aug. 1990.

[10] S. Rout and A. E. Bell, "Narrowing the performance gap between orthogonal and biorthogonal wavelets," in Conf. Rec. 38th Asilomar Conf. Signals, Syst. Comput. 2004, vol. 2, pp. 1757-1761.

[11] Martin vetterli,"A theory of multirate filter banks,'IEEE Trans. Acoust., Speech, Signal Process., vol. ASSP-35, no. 3, pp. 356372, Mar. 1987.

[12] James S Walker,'Wavelet Based Image Compression", Sub-chapter of CRC Press book: Transforms and Data Compression.

[13] J. Woods and S. O’Neil, "Subband coding of images," IEEE Trans.Acoust. Speech, Signal Process., vol. 34, no. 5, pp. 12781288 , Oct.1986.

[14] D. S. Taubman and M. W. Marcellin, JPEG2000: Image Compression Fundamentals. Norwell, MA: Kluwer, 2002, standards, and practice.

[15] B. E. Usevitch, “A tutorial on modern lossy wavelet image compression:Foundations of JPEG 2000,” IEEE Signal Process. Mag., vol. 18,no. 5, pp. 22-35, Sep. 2001.

[16] M. J. T. Smith and T. Barnwell, "Exact reconstruction techniques for tree-structured subband coders," IEEE Trans. Acoust. Speech, Signal Process., vol. ASSP-34, no. 3, pp. 434-441, Jun. 1986.

[17] S. Rout and A. E. Bell, "Narrowing the performance gap between orthogonal and biorthogonal wavelets," in Conf. Rec. 38th Asilomar Conf. Signals, Syst. Comput. 2004, vol. 2, pp. 1757-1761.

[18] Bradley j.Erickson M.D "Irrevesible compression of medical images" journal of digital imaging

[19] [19] Matthew J. Zukoski” A novel approach to medical image compression"Int. J. Bioinformatics Research and Applications, Vol. 2, No. 1, 200689

[20] David A. Clunie"Lossless Compression of Grayscale Medical Images - Effectiveness of Traditional and State of the Art Approaches"Quintiles Intelligent Imaging

[21] Yao-Tien Chen, Din-Chang Tseng"Wavelet-based medical image compression with adaptive prediction"sciencedirect.com

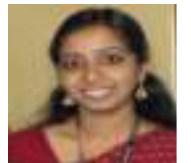

Anju B has completed her B.Tech degree in Computer Science and Engineering at St.Joseph's college of Engineering and Technology in the year 2011 and is currently doing M.Tech in the Department of Computer Science and Engineering, Karunya University, Coimbatore, India.Her specialization is in the area of image processing.

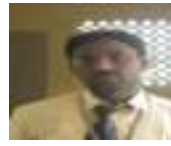

S. Manimurugan completed his Bachelor's Degree from Anna University and he received his Master's Degree from Karunya University. He was highly commended for his work in Image Processing and Information Security, for which he was honoured with a PhD from Anna University .His research interests Include Image Processing and Information Security. 